\title{
THE ASYMPTOTIC EXPANSION OF THE NUMBER OF TREE-LIKE POLYHEXES $\dagger$
}

\author{
by E. M. WRIGHT
}

(Received 28th September 1970)

We refer to (1) for the definitions of $U_{n}$ and $H_{n}$. Our object is to find asymptotic expansions for $U_{n}$ and $H_{n}$ for large $n$. This enables us to improve the approximations to $U_{n}$ and $H_{n}$ for large $n$ found in the last two pages of (1).

We write

$$
(1-x)^{k+\frac{1}{3}}=1+(-1)^{k-1} \sum_{n=1}^{\infty} c_{k, n} x^{n}
$$

so that

$$
c_{k, n}=\frac{(2 k+1) !(2 n-2 k-3) !}{2^{2 n-2} k !(n-k-2) ! n !} \sim \frac{\left(k+\frac{1}{2}\right)\left(k-\frac{1}{2}\right) \ldots \frac{1}{2}}{\pi^{\frac{1}{2}} n^{k+\frac{3}{2}}}
$$

for fixed $k$ and large $n$. Clearly

Also

$$
c_{k+1, n} / c_{k, n}=(2 k+3) /(2 n-2 k-3) \text {. }
$$

$$
(1-5 x)^{k+\frac{1}{2}}=1+(-1)^{k-1} \sum_{n=1}^{\infty} c_{k, n} 5^{n} x^{n} .
$$

Near $x=\frac{1}{5}$, we have

and so, if

$$
5^{k+\frac{1}{2}}(1-x)^{k+\frac{t}{t}}=(4+1-5 x)^{k+\frac{t}{t}}=2^{2 k+1} \sum_{t=0}^{\infty}\left(\begin{array}{c}
k+\frac{1}{2} \\
t
\end{array}\right) 2^{-2 t}(1-5 x)^{t}
$$

$$
\{(1-x)(1-5 x)\}^{k+\frac{k}{2}}=1+(-1)^{k-1} \sum_{n=1}^{\infty} d_{k, n} x^{n},
$$

we have, by Abel's result (see (2)),

$$
\begin{aligned}
d_{k, n} & =2^{2 k+1} 5^{n-k-\frac{1}{t}}\left\{\sum_{t=0}^{T-1}(-1)^{t}\left(\begin{array}{c}
k+\frac{1}{2} \\
t
\end{array}\right) 2^{-2 t} c_{k+t, n}+O\left(c_{k+T, n}\right)\right\} \\
& =2^{2 k+1} 5^{n-k-\frac{1}{t}} c_{k, n}\left\{\sum_{t=0}^{T-1}(-1)^{t} \frac{\left(k+t+\frac{1}{2}\right) \ldots\left(k-t+\frac{3}{2}\right) 2^{-2 t}}{t !\left(n-k-\frac{3}{2}\right) \ldots\left(n-k-t-\frac{1}{2}\right)}+O\left(n^{-T}\right)\right\} .
\end{aligned}
$$

$\dagger$ The research reported herein has been sponsored in part bv the United States Government. 
It follows from (9) of (1) that

$$
U_{n}=\frac{1}{2} d_{0, n+1}=5^{n+\frac{t}{2}} c_{0, n+1}\left\{\sum_{t=0}^{T-1}(-1)^{t} \frac{\left(t+\frac{1}{2}\right) \ldots\left(-t+\frac{3}{2}\right) 2^{-2 t}}{t !\left(n-\frac{1}{2}\right) \ldots\left(n-t+\frac{1}{2}\right)}+O\left(n^{-T}\right)\right\}
$$

and from (17) of (1) that

$$
\begin{aligned}
H_{n} & =\frac{1}{24} d_{1, n+2}+O\left(5^{\frac{1}{2} n}\right) \\
& =\frac{1}{3} 5^{n+\frac{1}{2}} c_{1, n+2}\left\{\sum_{t=0}^{T-1}(-1)^{t} \frac{\left(t+\frac{3}{2}\right) \ldots\left(-t+\frac{5}{2}\right) 2^{-2 t}}{t !\left(n-\frac{1}{2}\right) \ldots\left(n-t+\frac{1}{2}\right)}+O\left(n^{-T}\right)\right\} .
\end{aligned}
$$

These are the asymptotic expansions of $U_{n}$ and $H_{n}$ for large $n$. Harary and Read (1) show that

$$
U_{n} /\left(H_{n}(n+2)\right) \rightarrow 2
$$

as $n \rightarrow \infty$. From the above, the left hand side of (2) is $2+O(1 / n)$. Using the first two terms in our asymptotic expansions for $U_{n}$ and $H_{n}$, we find that

$$
U_{n} /\left\{H_{n}(n+2 \cdot 75)\right\}=2+O\left(n^{-2}\right) .
$$

Taking Harary and Read's example (and using their numerical results) we have which verifies (3).

$$
U_{40} /\left(42 \cdot 75 H_{40}\right)=2 \cdot 00281 \text {, }
$$

\section{REFERENCES}

(1) F. Harary and R. C. Read, The enumeration of tree-like polyhexes, Proc. Edinburgh Math. Soc. (2) 17 (1970), 1-13.

(2) E. C. Trtchmarsh, Theory of functions 2nd edition (Oxford, 1939), 224.

UNIVERSITY OF ABERDEEN 